\title{
Relação entre aptidão muscular e amplitude articular, por faixa etária, na marcha do idoso
}

\author{
Relationship between musde fitness and extent articular according to age group, \\ on the elderly'space

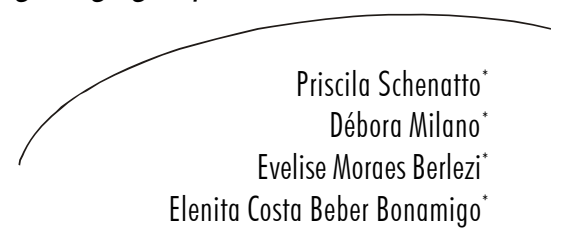

\section{Resumo}

Introdução: Com o aumento da longevidade, estudos realizados com pessoas idosas vêm despertando cada vez mais o interesse de pesquisadores de diversas áreas. É preciso compreender e analisar as mudanças relacionadas com o processo de envelhecimento e, através do esclarecimento destas, propiciar uma melhor qualidade de vida. Este estudo teve por objetivo identificar a associação das variáveis da aptidão muscular, amplitude e cadência de passos com a faixa etária. Materiais e métodos: A amostra foi constituída por 30 mulheres com idade acima de 65 anos, participantes dos grupos de convivência da Secretaria de Assistência Social do município de Ijuí/RS. Foram estudadas as seguintes variáveis: amplitude e cadência do passo, resistência muscular localizada de membros inferiores, flexibilidade de tronco, quadril, joelho e tornozelo e força muscular de membros inferiores. Respectivamente, essas variáveis foram avaliadas pelos seguintes métodos: distância e tempo percorrido, marcha estacionária de dois minutos, goniometria e pressão dos membros inferiores sobre a balança. As variáveis foram comparadas nas diferentes faixas etárias. Resultados: Os resultados obtidos com a pesquisa foram: quanto à flexão de tronco, a média geral obtida da amostra foi de 90,67 $( \pm 8,27)$ graus. Em relação à flexão de quadril, a média obtida foi de 105,83 $( \pm 9,83)$ graus; na extensão do quadril, a média foi de $9,47( \pm 0,81)$. $\mathrm{Na}$ análise da flexibilidade do joelho, observou-se que a média geral para o movimento de flexão foi de 111,17 $( \pm 10,31)$ graus; no movimento de extensão, a média foi de 0,87 $( \pm 1,59)$ graus. Na plantiflexão, a média geral da amplitude articular foi de $21,50( \pm 6,03)$ graus. Quando analisada a resistência muscular localiza-

Universidade Regional do Noroeste do Estado do Rio Grande do Sul, Departamento de Ciências da Saúde. Curso de Fisioterapia e Educação Física. Ijuí, RS, Brasil

Correspondência / Correspondence

Débora Milano

E-mail: d.milano@bol.com.br
Palavras-chave: Envelhecimento. Aptidão Física. Força Muscular. Amplitude de Movimento Articular. Marcha. Tono Muscular. Extremidade Inferior. Maleabilidade. Quadril. Joelho. Tornozelo. Qualidade de Vida. Mulheres. Amplitude de Passo. Cadência. 
da, a média geral do desempenho foi de 70,87 $( \pm 20,24)$ repetições No desempenho dos sujeitos quanto à força muscular de membros inferiores, a média geral do membro inferior direito foi de 15,06 $( \pm 4,20)$, e membro inferior esquerdo, 15,67 ( $\pm 6,86)$. Quanto a amplitude e cadência do passo, a média total da amplitude do passo entre os sujeitos é de $0,56( \pm 0,14) \mathrm{cm}$; já na cadência de passo, a média geral é $1,49( \pm 0,27)$ passo/segundos. Conclusão: A análise dos dados sugere que há associação entre as variáveis da aptidão muscular com a faixa etária, sendo que estas têm forte influência sobre a marcha. Com o avanço da idade, a cadência de passos tende a aumentar e a amplitude, a diminuir.

\section{Abstract}

Introduction: With increased longevity, more and more studies on the elderly interest researchers in different areas. We must understand and analyze the changes related to the aging process and, in making them clear, provide a better quality of life. Objective: This study aimed to check the association of the variables muscular aptitude, amplitude and cadence of steps with age group. Materials and methods: The sample comprised 30 women aged over 65 years, from activity groups at the Social Assistance Secretariat in the city of Ijuí/RS. The following variables were studied: amplitude and cadence of the step, located muscular resistance of inferior members, trunk flexibility, hip, knee and ankle and muscular force of inferior members. Respectively, these variables had been evaluated by the following methods: distance and covered time, 2-minute stationary pace, goniometry and pressure of inferior members on the scale. The variables were compared in the different age groups. Results: Research outcomes are the following: for trunk flexion, general average of the sample was $90.67( \pm 8.27)$ degrees. In relation to hip flexion, average was $105.83( \pm 9.83)$ degrees; for hip extension, average was $9.47( \pm 0.81)$. In the analysis of knee flexibility, the general average for the flexion movement was $111.17( \pm 10.31)$ degrees; for extension movement, it was $0.87( \pm 1.59)$ degrees. For plantiflexion, the general average of articular amplitude was $21.50( \pm 6.03)$. When resistance located was analyzed, general average of the performance was $70.87( \pm 20.24)$ repetitions. In the performance of individuals concerning muscular force of inferior members, general average of the right inferior member was $15.06( \pm 4.20)$, and left inferior member, $15.67( \pm 6.86)$. Concerning amplitude and step cadence, the total average of the step amplitude among the elderly was $0.56( \pm 0.14)$ $\mathrm{cm}$, and in the step cadence, general average was $1.49( \pm 0.27)$ step/seconds. There was statistical difference when step cadence was analyzed as compared to age group. Conclusion: Data analysis suggested association between the variable muscular aptitude and age group, but it has strong influence on pace. With aging, steps cadende tends to increase, and amplitude, to reduce.
Key words: Aging. Physical Fitness. Muscle Strength. Range of Motion Articular. Gait. Muscle Tônus. Lower Extremity. Hip. Knee. Ankle. Quality of life. Women. Pliability. 
INTRODUÇÃO

O envelhecimento é um processo no qual ocorrem mudanças morfofuncionais ao longo da vida, que começam após a maturação sexual e comprometem progressivamente a capacidade de resposta dos indivíduos ao estresse ambiental e à manutenção da homeostasia.

O sedentarismo, a incapacidade e a dependência são importantes adversidades à saúde que, associadas ao envelhecimento, contribuem para a perda de autonomia. De acordo com o Centro Nacional de Estatística para a Saúde, ${ }^{1} 84 \%$ das pessoas com idade igual ou superior a 65 anos são dependentes para realizar suas atividades cotidianas. Estima-se que em 2020 ocorrerá aumento de 84 a 167\% no número de idosos com moderada ou grave incapacidade.

Ao longo do processo de envelhecimento, ocorrem muitas alterações micro e macroscópicas nas estruturas articulares dos esqueletos axial e apendicular, assim como no tecido periarticular. Entre as causas dessas alterações podem-se citar o envelhecimento, traumatismos, processos patológicos ou a combinação destes. Alterações - por exemplo, nos discos vertebrais, como perda da hidratação, aumento da rigidez e degeneração do colágeno - contribuem de forma significativa para a perda da amplitude do movimento ou da mobilidade da coluna vertebral. ${ }^{2}$

Com o envelhecimento, há uma diminuição lenta e progressiva da massa muscular, sendo o tecido nobre paulatinamente substituído por colágeno e gordura. A perda de massa muscular com o envelhecimento ("sarcopenia") tem sido demonstrada pela excreção da creatina urinária, que reflete o conteúdo de creatina nos músculos e a massa muscular total - com redução de aproximadamente $50 \%$ entre os 20 e 90 anos. $^{3}$

A atrofia das fibras observadas no músculo envelhecido inicia-se por volta dos 25 anos, com uma diminuição progressiva da área em cerca de $10 \%$ até perto dos 50 anos, progredindo para uma atrofia mais pronunciada, de tal modo que aos 80 anos o idoso sofre uma perda de cerca de $50 \%$ na área de secção transversal do músculo. ${ }^{4}$

O presente estudo se propôs a avaliar a relação da aptidão muscular e amplitude articular com a marcha do idoso, sendo consideradas quatro faixas etárias. Entendese que as variáveis força muscular, resistência muscular localizada e flexibilidade, na relação com a amplitude do passo e cadência, interferem na locomoção e qualidade de vida dos idosos.

\section{METODOLOGIA}

Esta pesquisa éum estudo transversal analítico-descritivo. A amostra foi constituída por 30 mulheres acima de 65 anos escolhidas de forma aleatória nos grupos. Os critérios de exclusão foram sujeitos com acometimentos neurológicos e com presença de déficits cognitivos.

Neste trabalho foram verificadas a associação das variáveis da aptidão muscular, ampli- 
tude articular e cadência de passos com a faixa etária, bem como a flexibilidade de tronco (flexores), quadril (flexão e extensão), joelho (flexão e extensão) e tornozelo (plantiflexores); verificaram-se a força e resistência muscular localizada de membros inferiores; e foram analisadas a amplitude da passada e a cadência de passos. Os resultados foram comparados em quatro diferentes faixas etárias.

Para a avaliação da marcha, ou seja, a amplitude e a cadência de passos no chão, foram demarcados traços, até completar 30 metros. O sujeito deveria manter a máxima velocidade de marcha (não sendo permitido correr). Anotou-se a distância percorrida medida do primeiro traço e o ponto que o calcanhar tocou ao final da execução do último passo, além do tempo gasto.

Para calcular a amplitude média do passo, foi feita a divisão da distância total percorrida pelo número de passos (32), ou seja, $\mathrm{AMP}=\mathrm{DTP} / 32$, que para adultos é de 1,41 metros, e cuja duração é em média 1,03s (6). Para a cadência, foi feita a relação entre o número total de passos e o tempo gasto em segundos, $\mathrm{CAD}=\mathrm{n}^{\circ}$ passos/tempo (7). Para adultos, a cadência média é de 113 passos/minuto ou 1,88 passos/s. ${ }^{5}$
Para a avaliação da resistência de membros inferiores, foi realizado o teste de marcha estacionária de dois minutos. ${ }^{6}$ Para o teste de força muscular, foi utilizada balança digital, posicionada em uma parede e o indivíduo em decúbito dorsal realizando movimento de extensão de joelho. As medidas de flexibilidade de tronco, quadril, joelho e tornozelo foram realizadas através da goniometria.

O estudo foi projetado de acordo com as Diretrizes e Normas Regulamentadoras de Pesquisa envolvendo Seres Humanos e foi submetido ao Comitê de Ética da Universidade Regional do Noroeste do Estado do Rio Grande do Sul (UNIJUI). Todos os sujeitos avaliados foram esclarecidos sobre o estudo e assinaram um termo de consentimento informado.

\section{RESULTADOS}

A população avaliada neste estudo foram 30 mulheres com idade superior a 65 anos $\mathrm{A}$ idade média das mulheres da amostra foi 73,13 anos $( \pm 5,64)$. De forma categorizada, a amostra está representada na tabela 1 . 
Tabela 1 - Distribuição da amostra por faixa etária. Ijuí, RS, 2007.

\begin{tabular}{lcc}
\hline Faixa etária & $\mathrm{N}$ & $\%$ \\
\hline 65 a 69 anos & 9 & 30 \\
70 a 74 anos & 8 & 26,6 \\
75 a 79 anos & 7 & 23,3 \\
+ de 80 anos & 6 & 20 \\
\hline
\end{tabular}

A tabela 2 mostra a distribuição da flexibilidade por faixa etária, a qual foi realizada a partir da quantificação da amplitude de movimento pelo método da goniometria. A tabela mostra a análise estatística nos aspectos de média, desvio padrão, intervalo de confiança e máximo e mínimo. A média apresentada por movimento é a expressão da média do lado direito e esquerdo, uma vez considerando-se que os sujeitos que constituíram a amostra não eram portadores de nenhuma doença articular limitante.

Quanto à flexão de tronco, a média obtida pelo grupo de 65 a 69 anos foi de $90( \pm$ 10) graus; nas faixas etárias 70 a 74,75 a 79 e com mais de 80 anos as médias foram, respectivamente: $96,88( \pm 7,03), 87,14( \pm 5,66)$ e $87,50( \pm 6,12)$. A média geral da amostra foi $90,67( \pm 8,27)$. Na comparação entre os grupos pelo método de LSD (menor diferença significativa), houve diferença estatística em relação à flexão de tronco entre os grupos 70 a 74 anos e 75 a 79 anos, com $\mathrm{p}=0,021$. Também houve diferença significativa ao se comparar o grupo de 70 a 74 anos com o grupo de 80 mais $(p=0,032)$.
Em relação à flexão de quadril, a média do grupo de 65 a 69 anos foi de 111,11 $( \pm 7,81)$ graus; na faixa etária de 70 a 74 anos, a média obtida foi de 103,75 $( \pm 11,87)$ graus; já no grupo com 75 a 79 anos, a média foi de $102,14( \pm 8,09)$ e no grupo com mais de 80 anos a média foi de 105,0 $( \pm 10,48)$ graus. Em relação ao total, a média obtida foi de 105,83 $( \pm 9,83)$ graus. $\mathrm{Na}$ análise dos dados não se verificou diferença estatisticamente significativa na comparação entre os grupos.

Quanto à extensão do quadril, a média total obtida foi de $9,47( \pm 0,81)$. Entre os grupos não foi verificada diferença importante dos graus articulares, e os testes estatísticos não apresentaram diferença.

$\mathrm{Na}$ análise da flexibilidade do joelho, observou-se que a média geral para o movimento de flexão foi de 111,17 ( $\pm 10,31$ ) graus; no movimento de extensão, a média geral foi de 0,87 $( \pm 1,59)$ graus. Na plantiflexão, a média geral da amplitude articular foi de $21,50( \pm 6,03)$, sem diferença estatística entre os grupos. 
Tabela 2 - Estatística descritiva da flexibilidade por faixa etária. Ijuí, RS, 2007.

\begin{tabular}{|c|c|c|c|c|c|c|c|c|}
\hline & & $\mathrm{N}$ & Média & Desvio $p$ & $\begin{array}{r}\text { Intervalo } \\
95 \%\end{array}$ & $\begin{array}{l}\text { Oonfiança de } \\
\text { Média. }\end{array}$ & Mín & Máx \\
\hline & & & & & Limite Inf & Limite Sup & & \\
\hline Flex $\mathrm{t}$ & 65 a 69 anos & 9 & 90,00 & 10,000 & 82,31 & 97,69 & 70 & 100 \\
\hline & 70 a 74 anos & 8 & 96,88 & 7,039 & 90,99 & 102,76 & 90 & 110 \\
\hline & 75 a 79 anos & 7 & 87,14 & 5,669 & 81,90 & 92,39 & 80 & 95 \\
\hline & 80 anos ou mais & 6 & 87,50 & 6,124 & 81,07 & 93,93 & 80 & 95 \\
\hline & Total & 30 & 90,67 & 8,277 & 87,58 & 93,76 & 70 & 110 \\
\hline Flex q & 65 a 69 anos & 9 & 111,11 & 7,817 & 105,10 & 117,12 & 100 & 120 \\
\hline & 70 a 74 anos & 8 & 103,75 & 11,877 & 93,82 & 113,68 & 90 & 120 \\
\hline & 75 a 79 anos & 7 & 102,14 & 8,092 & 94,66 & 109,63 & 90 & 110 \\
\hline & 80 anos ou mais & 6 & 105,00 & 10,488 & 93,99 & 116,01 & 90 & 120 \\
\hline & Total & 30 & 105,83 & 9,833 & 102,16 & 109,51 & 90 & 120 \\
\hline Ext q & 65 a 69 anos & 9 & 9,78 & ,441 & 9,44 & 10,12 & 9 & 10 \\
\hline & 70 a 74 anos & 8 & 9,38 & ,916 & 8,61 & 10,14 & 8 & 10 \\
\hline & 75 a 79 anos & 7 & 9,43 & ,976 & 8,53 & 10,33 & 8 & 10 \\
\hline & 80 anos ou mais & 6 & 9,17 & ,983 & 8,13 & 10,20 & 8 & 10 \\
\hline & Total & 30 & 9,47 & ,819 & 9,16 & 9,77 & 8 & 10 \\
\hline Flex j & 65 a 69 anos & 9 & 114,44 & 5,270 & 110,39 & 118,50 & 110 & 120 \\
\hline & 70 a 74 anos & 8 & 110,00 & 14,142 & 98,18 & 121,82 & 90 & 130 \\
\hline & 75 a 79 anos & 7 & 105,00 & 8,660 & 96,99 & 113,01 & 95 & 120 \\
\hline & 80 anos ou mais & 6 & 115,00 & 10,488 & 103,99 & 126,01 & 100 & 130 \\
\hline & Total & 30 & 111,17 & 10,313 & 107,32 & 115,02 & 90 & 130 \\
\hline Ext j & 65 a 69 anos & 9 & ,22 & ,667 &,- 29 &, 73 & 0 & 2 \\
\hline & 70 a 74 anos & 8 & ,63 & 1,188 &,- 37 & 1,62 & 0 & 3 \\
\hline & 75 a 79 anos & 7 & 1,43 & 1,988 &,- 41 & 3,27 & 0 & 5 \\
\hline & 80 anos ou mais & 6 & 1,50 & 2,345 & 96 & 3,96 & 0 & 5 \\
\hline & Total & 30 & ,87 & 1,592 & ,27 & 1,46 & 0 & 5 \\
\hline Plant & 65 a 69 anos & 9 & 21,67 & 5,590 & 17,37 & 25,96 & 15 & 30 \\
\hline & 70 a 74 anos & 8 & 23,75 & 7,440 & 17,53 & 29,97 & 10 & 35 \\
\hline & 75 a 79 anos & 7 & 20,00 & 4,082 & 16,22 & 23,78 & 15 & 25 \\
\hline & 80 anos ou mais & 6 & 20,00 & 7,071 & 12,58 & 27,42 & 10 & 30 \\
\hline & Total & 30 & 21,50 & 6,039 & 19,25 & 23,75 & 10 & 35 \\
\hline
\end{tabular}


Na tabela 3, verificamos o desempenho dos participantes quanto à resistência muscular localizada de membros inferiores, apresentada segundo a distribuição por faixa etária. No grupo com idade de 65 a 69 anos, a média obtida foi de $71( \pm 22,53)$ repetições em dois minutos; para os grupos com idade de 70 a 74,75 a 79 e com mais de 80 anos, as médias verificadas foram, respectivamente: $79,50( \pm 17,42), 69,29( \pm 18,48)$ e $61( \pm 22,12)$. A média geral do desempenho $70,87( \pm 20,24)$ repetições.

Quando comparamos os grupos, verificamos diferença no número de repetições: o grupo de 65 a 69 anos tem desempenho superior ao grupo com mais de 80 anos. Os dados sugerem que, com o avanço da idade, o desempenho na prova da marcha estacionário diminui, mas, estatisticamente, a diferença entre os grupos não mostrou significância.

Tabela 3 - Estatística descritiva da resistência muscular localizada por faixa etária. Ijuí, RS, 2007.

\begin{tabular}{cccccccc}
\hline & & & \multicolumn{5}{c}{ Intervalo de Confiança } \\
& N & Média & Desvio p & de 95\% p/ a Média. & Mín & Máx \\
\hline 65 a 69 anos & 9 & 71,00 & 22,533 & 53,68 & 88,32 & 44 & 125 \\
70 a 74 anos & 8 & 79,50 & 17,427 & 64,93 & 94,07 & 54 & 106 \\
75 a 79 anos & 7 & 69,29 & 18,482 & 52,19 & 86,38 & 43 & 100 \\
$\begin{array}{c}\text { 80 anos ou } \\
\text { mais }\end{array}$ & 6 & 61,00 & 22,127 & 37,78 & 84,22 & 28 & 90 \\
$\quad$ Total & 30 & 70,87 & 20,243 & 63,31 & 78,43 & 28 & 125 \\
\hline
\end{tabular}

A tabela 4 demonstra o desempenho dos sujeitos do estudo quanto à força muscular de membros inferiores. Observa-se que a média para o membro inferior direito, obtida pelo grupo de 65 a 69 anos, foi de 15,64 $( \pm 4,81) \mathrm{Kg}$, e que, respectivamente, as médias para os grupos de 70 a 74 anos, 75 a 79 anos e com mais de 80 anos foram: 12,28 $( \pm 3,85) \mathrm{Kg}, 17,0( \pm 2,92) \mathrm{Kg}$ e $15,61( \pm 3,94)$ $\mathrm{Kg}$. A média geral foi de 15,06 $( \pm 4,20)$.

Para o membro inferior esquerdo, as médias respectivas para os grupos categorizados foram: $17,50( \pm 5,28), 10,81( \pm 1,75)$,
$18,44( \pm 6,97)$ e $16,16( \pm 10,51)$. A média geral foi de $15,67( \pm 6,86)$.

$\mathrm{Na}$ análise estatística, comparando-se os grupos, verificou-se diferença significativa para a força muscular do membro inferior direito entre o grupo de 70 a 74 anos e 75 a 79 anos, com $p=0,032$. Também para o lado esquerdo, observou-se diferença estatística quando se comparou o grupo de 65 a 69 anos com o de 70 a 74 anos $(p=0,043)$. Ainda houve diferença entre o grupo de 70 a 74 anos, comparado ao de 75 a 79 anos $(p=0,031)$. 
Tabela 4 - Estatística descritiva da força muscular por faixa etária. Ijuí, RS, 2007.

\begin{tabular}{|c|c|c|c|c|c|c|c|c|}
\hline & $\begin{array}{l}\text { Faixa } \\
\text { Etária }\end{array}$ & $\mathrm{N}$ & Média & Desvio $\mathrm{p}$ & $\begin{array}{c}\text { Intervalo de } \\
\text { Confiança de } 95 \% \\
\text { para a Média. }\end{array}$ & Mínimo & Máximo & $\mathrm{N}$ \\
\hline \multirow[t]{5}{*}{${ }^{*} \mathrm{MD}$} & $\begin{array}{c}65 \text { a } 69 \\
\text { anos }\end{array}$ & 9 & 15,644 & 4,8180 & 11,941 & 19,348 & 8,9 & 22,5 \\
\hline & $\begin{array}{c}70 \text { a } 74 \\
\text { anos }\end{array}$ & 8 & 12,288 & 3,8576 & 9,062 & 15,513 & 5,7 & 16,2 \\
\hline & $\begin{array}{c}75 \text { a } 79 \\
\text { anos }\end{array}$ & 7 & 17,000 & 2,9229 & 14,297 & 19,703 & 12,3 & 20,0 \\
\hline & $\begin{array}{l}80 \text { anos } \\
\text { ou mais }\end{array}$ & 6 & 15,617 & 3,9433 & 11,478 & 19,755 & 10,3 & 22,0 \\
\hline & Total & 30 & 15,060 & 4,2004 & 13,492 & 16,628 & 5,7 & 22,5 \\
\hline \multirow[t]{5}{*}{${ }^{*} \mathrm{ME}$} & $\begin{array}{c}65 \text { a } 69 \\
\text { anos }\end{array}$ & 9 & 17,500 & 5,2898 & 13,434 & 21,566 & 9,7 & 27,7 \\
\hline & $\begin{array}{c}70 \text { a } 74 \\
\text { anos }\end{array}$ & 8 & 10,812 & 1,7570 & 9,344 & 12,281 & 9,2 & 14,0 \\
\hline & $\begin{array}{c}75 \text { a } 79 \\
\text { anos }\end{array}$ & 7 & 18,443 & 6,9754 & 11,992 & 24,894 & 10,8 & 32,0 \\
\hline & $\begin{array}{l}80 \text { anos } \\
\text { ou mais }\end{array}$ & 6 & 16,167 & 10,5183 & 5,128 & 27,205 & 2,4 & 31,0 \\
\hline & Total & 30 & 15,670 & 6,8601 & 13,108 & 18,232 & 2,4 & 32,0 \\
\hline
\end{tabular}

*MD: membro direito, ME: membro esquerdo.

A tabela 5 demonstra o desempenho dos sujeitos do estudo quanto a amplitude e cadência de passo. Observa-se que a média total da amplitude do passo entre os sujeitos é de $0,56( \pm 0,14) \mathrm{cm}$; já na cadência de passo, a média geral é 1,49 $( \pm 0,27)$ passo/segundos. 
Tabela 5 - Estatística descritiva da amplitude e cadência de passo por faixa etária. Ijuí, RS, 2007.

\begin{tabular}{|c|c|c|c|c|c|c|c|c|}
\hline & & \multirow[t]{2}{*}{$\mathrm{N}$} & \multirow[t]{2}{*}{ Média } & \multirow[t]{2}{*}{ Desvio $\mathrm{p}$} & \multicolumn{2}{|c|}{$\begin{array}{l}\text { Intervalo de Confiança de } \\
95 \% \text { para a Média. }\end{array}$} & \multirow[t]{2}{*}{ Mínimo } & \multirow[t]{2}{*}{ Máximo } \\
\hline & & & & & $\begin{array}{l}\text { Limite } \\
\text { Inferior }\end{array}$ & $\begin{array}{l}\text { Limite } \\
\text { Superior }\end{array}$ & & \\
\hline \multirow[t]{5}{*}{ AMP } & 65 a 69 anos & 9 & ,6178 & , 17006 & ,4871 &, 7485 &, 48 & ,93 \\
\hline & 70 a 74 anos & 8 &, 5213 & ,02997 & ,4962 &, 5463 & ,48 &, 58 \\
\hline & 75 a 79 anos & 7 &, 5571 & , 13768 & ,4298 & ,6845 & ,38 & ,82 \\
\hline & $\begin{array}{l}80 \text { anos ou } \\
\text { mais }\end{array}$ & 6 &, 5367 & ,21370 &, 3124 & ,7609 & ,33 & ,93 \\
\hline & Total & 30 &, 5617 & , 14681 &, 5068 & ,6165 & ,33 & ,93 \\
\hline \multirow[t]{5}{*}{ CAD } & 65 a 69 anos & 9 & 1,5744 & , 15985 & 1,4516 & 1,6973 & 1,28 & 1,77 \\
\hline & 70 a 74 anos & 8 & 1,7163 & , 18118 & 1,5648 & 1,8677 & 1,33 & 1,88 \\
\hline & 75 a 79 anos & 7 & 1,3543 & ,31410 & 1,0638 & 1,6448 & 1,04 & 2,00 \\
\hline & $\begin{array}{l}80 \text { anos ou } \\
\text { mais }\end{array}$ & 6 & 1,2500 &, 17470 & 1,0667 & 1,4333 & 1,03 & 1,45 \\
\hline & Total & 30 & 1,4960 & ,27075 & 1,3949 & 1,5971 & 1,03 & 2,00 \\
\hline
\end{tabular}

$\mathrm{Na}$ análise da marcha, verificou-se diferença estatística ao se analisar a cadência de passos associada à faixa etária. Quando comparado o grupo de 65 a 69 anos com o grupo de 80 anos ou mais, obteve-se $\mathrm{p}=0,008$. Também houve diferença estatística na comparação do grupo de 70 a 74 anos com o grupo de 75 a 79 anos $(p=0,003)$, observando-se significância estatística entre o grupo de 70 a 74 anos com o de 80 anos $(p=0,000)$. A diferença média é significativa a um nível de 0,01 para estas associações.

\section{DISCUSSÃO}

Este estudo teve por objetivo analisar o desempenho físico de mulheres acima de 65 anos, nas variáveis que influenciam na marcha, particularmente a amplitude articular (tronco, quadril, joelho e tornozelo), força muscular e resistência muscular localizada, amplitude de passo e cadência do passo. Foi avaliada a flexibilidade do tronco, quadril, joelho e tornozelo.

Os dados obtidos sugerem que as variáveis do estudo sofrem influência com a idade, tanto em uma análise individual dos aspectos físicos (amplitude articular, força e resistência), como na análise da amplitude e cadência de passo, que tem associação direta com os componentes da aptidão muscular.

Fiatarone et al. ${ }^{7}$ constataram que a força muscular e a velocidade de marcha em ido- 
sos com idade avançada (entre 87 e 90 anos) aprimoraram-se cerca de $113 \%$ e $12 \%$, respectivamente, após um treinamento de resistência de 10 semanas com intensidade de $80 \%$ da força máxima. Na mesma linha, Ades et al. ${ }^{8}$ demonstraram que três meses de treinamento com pesos resultaram em incremento da capacidade submáxima de marcha ( $80 \%$ do pico de consumo de oxigênio) em indivíduos de 65 a 79 anos de idade. Aqueles que treinaram melhoraram seu tempo em nove minutos (de $25 \pm 4$ min para 34 $\pm 9 \mathrm{~min})$, representando um ganho de $38 \%$. Lord et al. ${ }^{9}$ também identificaram melhorias significativas na velocidade habitual de marcha em idosos, após 22 semanas de treinamento envolvendo atividades de força, equilíbrio e flexibilidade.

Neste contexto, a alteração funcional como a marcha fica visível em sujeitos com declínio importante da amplitude, força muscular e resistência muscular localizada. Se há limitação da amplitude articular de tronco, quadril, joelho ou tornozelo, a amplitude do passo fica comprometida, e o mesmo ocorre quando há déficit da força muscular. Associando-se o declínio da amplitude articular com a força muscular, a cadência de passo fica comprometida.

Alguns autores classificam a velocidade média da marcha em homens como $100 \mathrm{a}$ 120 passos por minuto e, para mulheres, 105 a 125 passos por minuto. Frequências acima ou abaixo destes valores são classificadas como velocidades de marchas rápidas ou lentas. ${ }^{10}$ As alterações na velocidade da marcha são feitas por vários fatores, tais como alterações do comprimento da passada ou da cadência e, usualmente, o indivíduo altera ambos os parâmetros. ${ }^{11}$

As evidências indicam que idosos caminham mais lentamente que jovens. Isso ocorre, principalmente, em virtude de uma redução da amplitude das passadas, associada a um declínio da flexibilidade de quadril e tornozelos e da força dos músculos flexores do tornozelo e dos extensores e flexores do joelho. Isso é compensado pelo aumento da cadência e do tempo da fase de apoio do passo, ainda que com um contato mais curto do calcanhar com o solo.

Pesquisas publicaram extensa análise da gênese das instabilidades da marcha, que foi feita junto a um grupo de 67 homens e mulheres com mais de 70 anos de idade. ${ }^{12}$ Além de investigar as limitações que poderiam estar na origem dos comprometimentos constatados, foi aplicado um programa de treinamento doméstico de seis meses, em delineamento randomizado controlado, visando ao desenvolvimento da força muscular e do equilíbrio. Os autores chegaram à conclusão de que as razões para as mudanças no padrão de marcha são muitas, mas que o incremento na função muscular de membros inferiores pode contribuir decisivamente para reduzir a instabilidade deambulatória. Nesse aspecto, alguns autores relataram que, em comparação com sujeitos mais jovens, os idosos adotam um padrão mais conservador de marcha, caracterizado por uma velocidade reduzida, menor amplitude de passo e maior variabili- 
dade de frequência, o que tende a se agravar em superfícies irregulares. ${ }^{13}$ Dentre os fatores que contribuem para a adoção desse padrão, os autores deram especial destaque à redução da força muscular dos membros inferiores.

Estudo realizado por Hausdorff et al. ${ }^{12}$ com 230 mulheres com mais de 75 anos constatou que déficits de força nos movimentos de extensão e flexão do joelho e tornozelo estariam mais associados ao desempenho na marcha do que ao próprio equilíbrio estático ou dinâmico. Outros autores chegaram a conclusões semelhantes a esse estudo, no que diz respeito ao padrão de marcha em idosos, mas relataram que o impacto dos movimentos de tornozelo (flexão e extensão), tanto por déficits de força quanto de flexibilidade, teriam maior influência na amplitude do passo do que os associados aos movimentos do joelho ou quadril. ${ }^{14}$

Durante a vida ativa, adultos perdem em torno de 8 a 10 centímetros de flexibilidade na região lombar e no quadril, quando medida por meio do teste de alcance máximo. Dentre os vários fatores que colaboram para isso estão a maior rigidez de tendões, ligamentos e cápsulas articulares, devido a deficiências no colágeno. ${ }^{15}$

Apesar de comprovado por inúmeros estudos que a atividade física minimiza os declínios do envelhecimento, o sedentarismo tem aumentado muito na atualidade, contribuindo para acelerar as perdas funcionais no idoso. ${ }^{16}$

\section{CONCLUSÃO}

As mudanças que ocorrem na marcha do idoso, como a literatura salienta, estão relacionadas às alterações do sistema músculoesquelético, o qual é passível de adaptação através de treinamento que respeite especificidades. Mesmo havendo a possibilidade do treinamento para retardar as alterações, inúmeros idosos têm sua independência funcional comprometida por elas.

A perda considerável da força muscular dos membros inferiores se reflete na dificuldade do idoso em se deslocar por uma distância maior, atravessar uma rua, subir lances de escada, entre outras situações não menos relevantes e que culminam no isolamento social. Obviamente, as alterações fisiológicas que ocorrem no processo de envelhecimento têm correlação com o estilo de vida adotado pelo indivíduo.Assim, um idoso que pratica atividade física sofre declínio das suas aptidões, porém comparado a um sujeito que não pratica atividade física, o declínio é inferior.

As evidências indicam que idosos caminham mais lentamente que jovens. Isso ocorre, principalmente, em virtude de uma redução da amplitude das passadas, associada a um declínio da flexibilidade de quadril e tornozelos e da força dos músculos flexores do tornozelo e dos extensores e flexores do joelho. ${ }^{16}$

Os dados obtidos neste estudo sugerem que as variáveis do estudo sofrem influência com a idade, tanto em uma aná- 
lise individual dos aspectos físicos (amplitude articular, força e resistência) como na análise da amplitude e cadência de passo, que tem associação direta com os componentes da aptidão muscular. Isso comprova que, com o avanço da idade, a cadência de passos tende a aumentar, e a amplitude, a diminuir.

\section{REFERÊNCIAS}

1. Matsudo SMM, Matsudo VKR, Barros Neto TL. Impacto de envelhecimento nas variáveis antropométricas, neuromotoras e metabólicas da aptidão física. Revista Brasileira de Ciência \& Movimento 2000 set; 8(4): 21-32.

2. Amundsen LR. Efeitos de envelhecimento nas articulações nos ligamentos. In: Kauffman T. Manual de reabilitação geriátrica. 1. ed. Rio de Janeiro: Guanabara Koogan; 2001. p. 12-24.

3. Rossi E, Sader CS. Envelhecimento do sistema osteoarticular. In: Freitas EV, et al. Tratado de Geriatria e Gerontologia. 1.ed. Rio de Janeiro: Guanabara Koogan; 2002. p. 508-14.

4. Lexell J, Taylor C, Sjöström M. What is the cause of the ageing atrophy? Total number, size and proportion of different fiber types studied in whole vastus lateralis muscle from 15- to 83-year-old men. J Neurol Sci 1988 apr; 84(2-3): 275-94.

5. Barela AMF. Análise biomecânica do andar de adultos e idosos nos ambientes aquático e terrestre. [Tese]. São Paulo; Universidade de São Paulo; 2005.

6. Farinati PTV, Lopes LNC. Amplitude e cadência do passo e componentes da aptidão muscular em idosos: um estudo
Fica aqui a sugestão para que novos estudos sejam realizados, tanto de avaliação das condições físicas e funcionais do idoso, para a identificação dos problemas, como para o desenvolvimento de programas que possam intervir, de forma positiva, na aptidão muscular desses sujeitos, buscando manter ou melhorar a potência no desempenho de atividades funcionais, tais como a marcha.

correlacional multivariado. Revista

Brasileira de Medicina do Esporte 2004; 10(5): 389-94.

7. Fiatarone MA, et al. High intensity strength training in nonagenarians: effects on skeletal muscle. JAMA 1990; 263(22): 3029-34.

8. Ades PA, et al. Weight training improves walking endurance in healthy elderly persons. Ann Intern Med 1996 mar 15; 124(6): 568-72.

9. Lord SR, et al. The effect of exercise on gait patterns in older women: a randomized controlled trial. J Gerontol A Biol Sci Med Sci 1996, 51(2): M64-70.

10. Pickles B, at al. Fisioterapia na $3^{a}$ idade. São Paulo: Livraria Santos; 2000.

11. Hageman P. Treinamento da marcha. Revista da Fisioterapia, 2000

12. Hausdorff JM, et al. Etiology and modification of gait instability in older adults: a randomized controlled trial of exercise. J Appl Physiol 2001; 90(6): 211729.

13. Menz HB, Lord SR, Fitzpatrick RC. Agerelated differences in walking stability. Age Ageing 2003 mar; 32(2): 137-42. 
14. Judge JO, Davis 3rd RB, Ounpuu S. Step length reductions in advanced age: the role of ankle and hip kinetics. J Gerontol A Biol Sci Med Sci 1996 nov; 51(6): M303-12.

15. Shephard RJ. Aging and exercise. In: Encyclopedia of Sports Medicine and
Science. Fahey TD, editor. Internet Society for Sport Science, 2005.

16. Thomas SG. Programas de exercícios e atividades. In: Pickles B, et al. Fisiologia na $3^{\text {a }}$ Idade. 2. ed. São Paulo: Santos; 2000. p. $158-67$.

Recebido: $28 / 8 / 2008$

Revisado: 27/1/2009

Aprovado: 10/7/2009 